\title{
Neue Wege zu frischem Geld
}

VON BERTHOLD BECHER UND

JENS HAYER
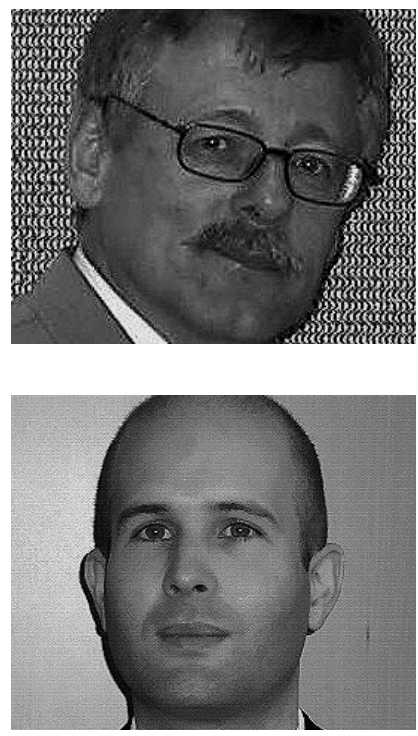

Dr. Berthold Becher und Jens Hayer sind Mitarbeiter der Bank für Sozialwirtschaft AG Internet http://www.sozialbank.de

\author{
Bankdarlehen werden für sozialwirtschaftliche Unter- \\ nehmen ein wichtiges Finanzierungsinstrument blei- \\ ben. Gleichzeitig etablieren sich jedoch weitere Mög- \\ lichkeiten, um Investitionen tätigen und neue \\ Geschäftskonzepte erproben zu können.
}

Die Finanzierung in der Sozial- und der Gesundheitswirtschaft befindet sich seit einigen Jahren in einem tiefgreifenden Veränderungsprozess. Diese Entwicklung ist nicht nur zurückzuführen auf die Finanzlage des Staates, der Sozialleistungs- und Sozialversicherungssysteme. Dahinter stehen ordnungspolitische Veränderungen wie Entstaatlichung, Verwettbewerblichung, neue Mechanismen der Leistungs- und Investitionsfinanzierung sowie zunehmende Kommerzialisierung von Sozialund Gesundheitsleistungen. Diese Entwicklungen werden in den kommenden Jahren - auch mit Verweis auf die fiskalischen Zwänge (1) - weiter vorangetrieben werden. Zugleich ist in vielen Leistungsbereichen ein steigender und sich differenzierender Bedarf auszumachen, der einen Aus- oder Umbau des Leistungsangebots und somit Investitionen nach sich zieht. Hinzu kommt, dass in einigen Bereichen (2) aktuell ein akuter Investitions- und Modernisierungsstau besteht.

\section{These 1: Die Unternehmen in der Sozial- und Gesundheitswirt- schaft müssen die Finanzierung auf eine breitere Basis stellen}

Die Unternehmen der Sozial- und der Gesundheitswirtschaft werden bei der Finanzierung zukünftig neben den abnehmenden Zuwendungen und den Vergütungen der Sozialleistungs- und
Sozialversicherungsträger vermehrt auf weitere Finanzierungsquellen zurückgreifen. Hier sind Einnahmen aus dem Leistungsverkauf an Nutzer sowie Nutzerkapital, ein verstärkter Einsatz von Eigenkapital, Kredit- und Kapitalmarktmittel, sozial gewidmetes Kapital sowie Einnahmen aus dem Leistungsverkauf an Unternehmen zu nennen (vgl. Übersicht »Finanzierungsquellen und -instrumente in der Sozial- und Gesundheitswirtschaft «, Seite 23).

\section{These 2: Die Ausgestaltung der Vergütung führt zu weiterem Effizienzdruck und zur Umgestaltung der Leistungserbringung}

Die Finanzierung von Leistungen und Investitionen über die Vergütungen der Sozialleistungs- und Sozialversicherungsträger wird schwieriger. Der Rationalisierungsdruck steigt aufgrund der Entwicklung der Vergütungshöhe und des zunehmenden Wettbewerbs. Es ist auch eine Wende von der einrichtungs- zur personenbezogenen Vergütung auszumachen: Die Vergütung wird individuell bedarfsorientiert ausgelegt und zudem mit dem Erfolg der Leistung verbunden.

Die Individualisierung der Vergütung und die Finanzierung bedarfsbestimmt differenzierter Leistungen erfordern von den Anbietern, fall- und personenbezogen Angebote zu ent- 
wickeln und flexibel anzubieten sowie eine differenziertere Kosten- und Ertragskalkulation und ein zeitnahes Controlling. Insbesondere wenn die Vergütung als Subjektfinanzierung gestaltet ist, also der Leistungsnutzer vom Finanzierungsträger mit Kaufkraft ausgestattet wird und als eigenverantwortlicher selbstbestimmender Nachfrager agiert, stehen die Sozial- und Gesundheitsunternehmen vor der Aufgabe, die Nachfrage auf sich zu ziehen und eine wettbewerbsfähige Preispolitik zu betreiben. Das bisherige Dreieck zwischen Leistungsträger, Leistungserbringer und Leistungsempfänger wird tendenziell aufgelöst.

Die Finanzierung über individualisierte Vergütungen ist aber nur bei den Leistungen sinnvoll, die eine individuelle Bedarfsfeststellung und Aufwandskalkulation vorab möglich ist; hiermit werden einer nicht bedarfsgebundenen Mengenausweitung Grenzen gesetzt (z. B. gewisse psychiatrische Hilfen und Obdachlosenhilfe). Bei Leistungen, deren Aufwand und Menge nicht vorab kalkulierbar sind, werden zunehmend Budgets eingeführt die auf Zielgruppen-, Sozialraum- oder Anbietergruppen kalkuliert sind.

\section{These 3: Der Anteil der Leistungen, die von den Nutzern teilweise oder zur Gänze zu finanzieren sind, wird sich vergrößern}

In der Sozial- und der Gesundheitswirtschaft bilden sich unterschiedliche Märkte heraus. Da ist zunächst einmal der Markt, in dem die Finanzierung der Leistungen vollständig über die Sozialleistungs- und die Sozialversicherungsträger erfolgt. Darüber hinaus erweitert sich der Markt, in dem die Leistungen ergänzend mit einem Eigenanteil der Nutzer finanziert werden. Auch der Markt, in dem Nutzer die Leistungen vollständig finanzieren, wird ebenfalls größer werden. Entsprechend der Gegebenheiten in den einzelnen Märkten, haben die Anbieter sich einer spezifischen einnahmenorientierten Leistungs- und Preispolitik zu bedienen.

In der Gesundheits- und der Sozialwirtschaft gewinnen die Eigenanteile und die Selbstzahlungen der Leistungsnutzer als Bestandteile der Leistungsfinanzierung zunehmend an Bedeutung. (3)
Hiermit wird vermehrt der Preis ein wichtiges Kriterium für die Inanspruchnahme eines Angebotes. Konsequenz dieser Entwicklung ist ein zunehmender Preiswettbewerb zwischen den Anbietern.

Die unmittelbare Einbindung von Nutzerkapital in die Investitionskostenfinanzierung gewinnt an Gewicht. Dies kann beispielsweise durch den Verkauf von Wohnrecht oder Wohneigentum erfolgen. (4) Beide Varianten setzten allerdings eine entsprechend finanziell ausgestattete Klientel voraus.

\section{These 4: Eine ausreichende Eigenkapitalausstattung wird in höherem Maße Voraussetzung für die Investitionsfähigkeit}

Um Investitionen selbst tätigen zu können oder den Zugang zu nichtöffentlichen Finanzierungsmitteln zu wettbewerbsfähigen Kosten zu erhalten, ist eine adäquate Eigenkapitalausstattung die Grundvorrausetzung. Je besser die Eigenkapitalquote, desto größer die Chance, Fremdmittel zu erhalten.

Eigenkapital wird auch benötigt, um neue innovative bedarfsgerechte und wettbewerbsbeständige Konzepte zu entwickeln und zu implementieren. Die Kosten hierfür werden nur selten von Finanzierungsträgern anerkannt oder mit Fördermitteln bezuschusst werden.

Um die nötigen Eigenmittel zu generieren, sind die Möglichkeiten der Innenfinanzierung auszuschöpfen. Dies kann durch Rationalisierung und vor allen Dingen durch eine einnahmenorientierte Leistungsentwicklung erfolgen. Allein das Reduzieren von Kosten bei gleichbleibenden Angeboten reicht nicht aus. Das Haften an traditionellen Leistungen muss ersetzt werden durch das pro-aktive und kreative Entwickeln von Leistungsangeboten für die Nutzer unmittelbar sowie für die Sozialleistungs- und Sozialversicherungsträger wie auch für andere Unternehmen.

Die Eigenkapitalbasis kann zudem erheblich verbessert werden, indem stille Reserven freigesetzt werden, beispielsweise durch den Verkauf von Grundstücken und Gebäuden.

Neben der Innenfinanzierung zur Generierung von Eigenkapital ist eine Eigenkapitalstärkung durch Außenfi- nanzierung zu nutzen. Hier handelt es sich größtenteils um Einlagen und Beteiligungen von Privatpersonen, Fonds, Stiftungen, Business Angels etc. Sofern Finanzierungen dieser Art verbunden sind mit Mitspracherechten der Mittelgeber steht der Gemeinnützigkeitsstatus einer Gewinnausschüttung entgegen.

\section{These 5: Die Angewiesenheit auf den Kredit- und Kapitalmarkt steigt}

In weiten Bereichen der Sozial- und der Gesundheitswirtschaft wird der Einsatz von Kredit- und Kapitalmarktmitteln zunehmend wichtiger. Im Mittelpunkt steht die Finanzierung von größeren Investitionen (z. B. Immobilien) oder von Unternehmenserweiterungen. Auch künftig werden Bankdarlehen ein essenzielles Finanzierungsinstrument für die Unternehmen bleiben. Darüber hinaus gewinnen jedoch weitere Instrumente des Kredit- und Kapitalmarktes wie Mezzanine-Kapital, Schuldscheindarlehen, Genusscheine, Anleihen und Aktien an Bedeutung.

In manchen Bereichen können klassische Bankdarlehen mit geförderten und zinsverbilligten Darlehen (z. B. der Kreditanstalt für Wiederaufbau oder den Förderbanken der Bundesländer) kombiniert und somit die Kapitalkosten reduziert werden. Diese Art der öffentlichen Förderung ist zumeist verbunden mit der Intention, die Entwicklungsrichtung des Leistungsangebots zu steuern.

Als kapitalschonende Finanzierungsformen haben sich in den letzten Jahren das Immobilien-und-MobilienLeasing sowie das Investor-BetreiberModell etabliert. Dies erlaubt es den Unternehmen, ihr Kapital im Kerngeschäft einzusetzen und die Herausforderungen eines Investors, großvolumige Immobilienfinanzierungen sicherzustellen, zu umgehen. (5) Als Investoren sind in diesem Bereich insbesondere geschlossene Fonds aktiv.

Zur Verbesserung der Bilanzstruktur und der Bonität sowie zur Erhöhung des Kreditspielraums können Unternehmen Mezzanine-Kapital aufnehmen. Eine Steigerung der Eigenkapitalausstattung kann hierbei ohne das Einräumen von Mitsprache- und Mitwirkungsrechten an die Investoren erfolgen. 
Die Attraktivität der Sozial- und Gesundheitswirtschaft für Financiers und Investoren ist vor allen Dingen begründet durch die relativ stabile Nachfrage aufgrund der Konjunkturunabhängigkeit, den langfristigen Zuwachs des Bedarfs in den verschiedenen Leistungsbereichen und die bisher weitgehende Unabhängigkeit von der individuellen Einkommens- und Vermögenssituation der Leistungsempfänger aufgrund der umlagefinanzierten Sozialversicherungssysteme.

Manche Unternehmensgründer und Kleinstunternehmen in der Sozial- und Gesundheitswirtschaft haben allerdings meist nur einen sehr geringen Kapitalbedarf und aufgrund fehlender Sicherheiten keinen direkten Zugang zu Bankkrediten. Infolgedessen wurden Mikrofinanz-Angebote entwickelt, welche auf internationaler Ebene schon länger verbreitet sind, in Deutschland aber erst allmählich zum Einsatz kommen.

Die Unternehmen in der Sozial- und Gesundheitswirtschaft waren bisher vor allen Dingen auf die öffentliche Hand als Finanzierungsquelle orientiert. Die Finanzierung über den Kredit- und Kapitalmarkt stellt andere Anforderungen an die Mittelnehmer. Die Mittelgeber stellen nicht einfach Kapital zur Verfügung, sondern sie finanzieren Zukunft. Daher reichen vergangenheitsbezogene Daten für die Finanzierungsentscheidung allein nicht aus.

Gefordert wird von den Mittelnehmern zumeist die plausible Darlegung des Geschäftsmodells, welches das Unternehmen zukünftig verfolgen will und mit dem es sich im Markt zu behaupten gedenkt. (7) Für das zu finanzierende Investment wird ein Businessplan verlangt. Dessen wesentlicher Bestandteil ist eine Planungsrechnung. Diese umfasst etwa die nächsten fünf Geschäftsjahre und hat die Marktentwicklung zu berücksichtigen, muss fachlich fundiert und mit einer soliden Finanzplanung unterlegt sein. Hierzu ist einschlägige Kompetenz im Management und Controlling von Nöten. Die Planungsrechnung quantifiziert das Investment in Form greifbarer Finanzzahlen. Für die Mittelgeber steht der Zahlungsüberschuss (»Cashflow «) für die Laufzeit der Finanzierung im Vordergrund. Für sie ist folgende Frage entscheidend: Ist der Mittelnehmer nachhaltig kapitaldienstfähig?

\section{These 6: Sozial gewidmetes Kapital gewinnt bei der Finanzierung von Innovationen an Bedeutung}

Zur Deckung des Finanzierungsbedarfs ist weitergehend sozial gewidmetes Kapital zu mobilisieren. Die Mittel werden von Privatpersonen, Unternehmen, Fördervereinen etc. zur Realisierung sozialer Zwecke bereitgestellt. Es kann unterschiedliche Erscheinungsformen haben wie direkte Spenden, Mittel aus Sozial- und Sparfonds (8) oder von Stiftungen sowie Anlagekapital.

Sozial gewidmetes Kapital wird somit nicht immer als »Zuwendung « gegeben, sondern kann mit der Erwartung an einen Return verbunden sein. Hier sind beispielsweise Dividendenzahlungen von gemeinnützigen Aktiengesellschaften an Wohlfahrtsvereinigungen zu nennen oder Renditen von Sozialunternehmen (9) ohne steuerrechtlichen Gemeinnützigkeitsstatus (10).

Sozial gewidmetes Kapital ist insbesondere auch für das Entwickeln und Realisieren von innovativen Konzepten und für Start-ups relevant.

Nicht nur die Finanzierungsträger und die Financiers des Kredit- und Kapitalmarktes, sondern auch die Mittelgeber von sozial gewidmetem Kapital legen verstärkt Wert auf die Auswahl des Verwendungszwecks der zur Verfügung gestellten Finanzmittel, auf die Effizienz der Mittel nehmenden Organisation, die Evaluation der finanzierten Maßnahme sowie auf Transparenz und regelmäßiges Reporting. Die Vergabe von Mitteln wird mit der Erwartung verbunden, dass das Investment eine möglichst hohe »soziale Rendite « erzielt, also möglichst viel »Gutes « bewirken soll. (11) Die Zeit, in der unhinterfragt Geld in soziale Projekte und Organisationen gegeben wurde, ist vorbei.

Die Maßnahmen zur Mobilisierung von sozial gewidmetem Kapital in seinen unterschiedlichen Ausprägungen werden weiter zu entwickeln sein. Es bedarf verbesserter institutioneller Vorkehrungen zur Vermittlung zwi- schen den unterschiedlichen (potentiellen) Kapitalgebern mit ihren spezifischen Interessen und Erwartungen einerseits und den sozialen Projekten und Organisationen andererseits. (12)

\section{These 7: Leistungsverkauf an andere Unternehmen wird zunehmend relevant}

Neben den dargestellten Finanzierungsinstrumenten nimmt in der Sozial- und der Gesundheitswirtschaft der Leistungsverkauf an Unternehmen aus anderen Wirtschaftsbereichen zu. Anbieter kooperieren beispielsweise mit einer Versicherung, die eine Haushaltsund Senioren-Unfallversicherung anbietet und erbringen befristete Assistenz-Leistungen im Schadensfall (13) und mit Unternehmen, die ihre Mitarbeiter in einer Pflegesituation unterstützen (14) oder ihren Mitarbeitern Kinderbetreuung anbieten. Auch hierdurch werden neue Einnahmequellen erschlossen.

\section{These 8: Auswahl und Kombination der Finanzierungsinstrumente gewinnen an Bedeutung}

Eine Finanzierung der Investitionen allein über das operative Geschäft ist meist nicht möglich. Daher müssen die sich bietenden Möglichkeiten der Außenfinanzierung genutzt werden. Die entsprechenden Finanzierungsinstrumente für die Sozial- und die Gesundheitswirtschaft werden vielfältiger.

Auszugehen von dem spezifischen Finanzierungsanlass (Start-up-Finanzierung, Investitionsfinanzierung, Wachstumsfinanzierung etc.), den eigenen wirtschaftlichen Voraussetzungen und der Rechtsform (z. B. Vereinbarkeit mit dem Gemeinnützigkeitsstatus) sind bei der Auswahl von Finanzierungsinstrumenten weiterhin Kriterien wie Volumen, Fristigkeit, Transaktionskosten zu berücksichtigen. Wichtig sind Mittelgeber, die kompetent sind in der Beurteilung der im Einzelfall geeigneten Finanzierungsinstrumente und die Sonderheiten des Marktes und der Branche kennen.

Die Finanzierungskosten sind wettbewerbsrelevant. Sie müssen bei den 
Leistungsnutzern und den Finanzierungsträgern durchgesetzt werden.

\section{These 9: Die Mittelnehmer müssen aktiv Einfluss auf die Rahmenbedingungen nehmen}

Die Bewältigung der Fiskalkrise sowie die erforderliche Umgestaltung der Angebotsstruktur fordern in höherem Maße von den Anbietern in der Sozialund der Gesundheitswirtschaft, proaktiv und neben den unmittelbaren Finanzierungsverhandlungen auf die Rahmenbedingungen der Finanzierung einzuwirken.

Ungeachtet des Rückzugs des Staates hat dieser doch - auch bei weiter gehender Kommerzialisierung und Vermarktlichung von Sozial- und Gesundheitsleistungen - eine Sicherstellungsverantwortung. Davon ausgehend sind von ihm ordnungspolitische Spielregeln zu setzen, die Sozialleistungs- und Sozialversicherungssysteme steuern sowie die Entwicklung des Leistungsangebots befördern. Daher sind Regelungen einzufordern, die eine nachhaltige Investitionsfinanzierung ermöglichen.

Die staatlichen Aktivitäten zur Bewältigung der Finanzmarkt- und Wirtschaftskrise haben gezeigt, wie wichtig es ist, auf die politische Prioritätensetzung Einfluss zu nehmen. Die anhaltenden Sparmaßnahmen in den öffentlichen Haushalten - verstärkt durch die Schuldenbremse im Grundgesetz -, aber auch die anstehenden ordnungspolitischen wie auch struktur- und leistungspolitischen Entscheidungen er-

\section{Anmerkungen}

(1) Durch die hohen Finanzierungsdefizite der öffentlichen Haushalte (erstes bis drittes Quartal 2010: 84,2 Milliarden Euro) wird der finanzielle Spielraum weiter eingeengt. Hinzu kommt, dass einer weiteren Auswei-

\section{"Die Zeit, in der unhinterfragt Geld in soziale Projekte und Organisationen gegeben wurde, ist vorbei"}

fordern die Einflussnahme der Akteure der Sozial- und Gesundheitswirtschaft zur Sicherung ihrer finanziellen Rahmenbedingungen. Hierbei ist $\mathrm{zu}$ berücksichtigen, dass die Interessenlagen der Akteure unterschiedlich und teilweise konträr sind.

Insgesamt gesehen können die Sozial- und die Gesundheitswirtschaft die in vielen Bereichen zunehmenden Bedarfe, die Folgekosten minimierende Effekte ihrer Leistungen wie auch ihre regionale- und volkswirtschaftliche Bedeutung in die Waagschale werfen.

\section{Finanzierungsquellen und -instrumente in der Sozial- und Gesundheitswirtschaft}

Es muss nicht immer ein Bankkredit sein: Sozialwirtschaftlichen Unternehmen steht mittlerweile eine breite $\mathrm{Pa}$ lette von Finanzierungsmöglichkeiten zurVerfügung.

\section{Öffentliche Mittel}

Investitionskostenzuschuss, Projektförderung etc.

\section{Vergütungen}

Grundsicherung im Alter, Hilfe zur Pflege, Betreuungs-, Gesundheits- und Pflegeleistungen, Eingliederungshilfe etc.

Einnahmen aus Leistungsverkauf an Nutzer und Nutzerkapital

Eigenanteil, Selbstzahlung, Wohnrechtsmodelle, Wohneigentumsmodelle etc.

\section{Eigenkapital}

Gewinne aus Leistungserbringung, Einlagen und Beteiligungen von Privatpersonen, Fonds, Stiftungen, Business Angels etc.

\section{Kredit- und Kapitalmarktmittel}

Investitionskredite, Mezzanine-Kapital, Schuldscheindarlehen, Anleihe, Immobilien- und Mobilien-Leasing, InvestorBetreiber-Modell, Mikrokredite etc.

\section{Sozial gewidmetes Kapital}

Gemeinschafts- und Bürgerstiftungen, Sozialfonds, Sparfonds, systematisches Fundraising, philanthropische Venture-Capital-Fonds etc.

Einnahmen aus Leistungsverkauf an Unternehmen

Einkaufsdienste, Fahrdienste, Begleitdienste, Kinderbetreuung, Beratung von pflegenden Angehörigen etc. tung der Verschuldung durch die sogenannte "Schuldenbremse « eine Grenze gesetzt wurde.

(2) So beispielsweise bei Pflegeheimen und bei Krankenhäusern. Experten beziffern den Sanierungsbedarf in den stationären Pflegeheimen mit rund sieben Milliarden Euro und den Investitionsstau bei Krankenhäusern mit rund 50 Milliarden Euro.

(3) Beispielsweise tragen die privaten Haushalte über 30 Prozent der gesamten Pflegeausgaben im Bereich des SGB XI.

(4) Beim Wohnrechtsmodell veräußert der Anbieter ein grundbuchlich gesichertes Wohnrecht in der Einrichtung gegen Zahlung eines Einmalbetrages. Im Gegensatz dazu erwerben potentielle Nutzerinnen und Nutzer beim Wohneigentumsmodell durch den Kauf einer Wohnung Eigentum an der Immobilie.

(5) Es darf jedoch nicht unterschätzt werden, dass weitere Investitionen nur mit Zustimmung der Eigentümer durchgeführt werden können, dass Investoren oft die Strategie haben, die Immobilien nach einigen Jahren gewinnbringend $\mathrm{zu}$ veräußern und dass Pachtverträge Veränderungen unterliegen können.

(6) Mit dem Start des Mikrofinanzfonds Deutschland Anfang 2010 soll jedoch mittel- bis langfristig ein flächendeckendes Mikrokreditangebot aufgebaut werden. Gemäß der Definition der Europäischen Kommission weisen Mikrokredite folgende Merkmale auf: Die Darlehenssumme ist kleiner als 25.000 Euro und die Kreditnehmer 
sind Kleinstunternehmer mit weniger als zehn Mitarbeitenden, einem Jahresumsatz kleiner als zwei Millionen Euro und einer Bilanzsumme unter zwei Millionen Euro.

(7) Das Geschäftsmodell umfasst den Nutzen, der gestiftet werden soll, die Leistungen, mit denen man Einnahmen erzielen will sowie die baulichen, personellen und betriebsorganisatorischen Voraussetzungen ihrer Erstellung.

(8) Sozialfonds generieren ihre Mittel aus einem Kapitalstock bestehend aus Geld- und Sacheinlagen (z. B. Grundstücke) unterschiedlicher Mittelgeber. Bei einem Sparfonds können die Mittelgeber wie bei einem banküblichen Sparbuch Gelder einzahlen, abheben und kündigen. Die Finanzmittel werden hierbei durch einen Zinsverzicht (»Sozialabschlag «) generiert.

(9) Als Sozialunternehmen wird jedes Unternehmen bezeichnet, dessen Handeln vor allen Dingen auf die Lösung von sozialen Problemen abzielt. Dies kann auch mit profitorientierten Zielen verbunden sein.

(10) Beispiel: Soziales Risikokapital und Wagniskapital von Privatinvestoren oder einem Fonds werden in Form von Eigenkapital oder Darlehen an ein Sozialunternehmen (z. B. einem Startup) vergeben. Da die finanzielle Rendite nicht im Vordergrund steht, sind die Erwartungen an diese deutlich geringer als in anderen Wirtschaftsbereichen.

(11) Ein Konzept, das sich mit der Bewertung von sozialen, ökonomischen und sozioökonomischen Leistungen sozialer Dienstleistungsorganisationen befasst, ist der Social Return on Investment (SROI).

(12) Ein Intermediär, der diese Funktion wahrnimmt, ist beispielsweise die PHINEO gAG. Die Arbeitsweise und die Effektivität sozialer Projekte werden von PHINEO anhand einer mehrstufigen Analyse bewertet. Die Ergebnisse werden sozialen Investoren aber auch Vermögensberatern auf einer Plattform zur Verfügung gestellt.

(13) Beispiele: Besuchsdienste, Einkaufsdienste, Fahrdienste, Putzdienste, Begleitdienste, Reinigung, Wäscheversorgung, Menüservice, Körperpflege.

(14) Beispiele: das Informieren der Betroffenen, eine Pflegehotline, Schulungsmaßnahmen und die Vermittlung weiterer Hilfen.
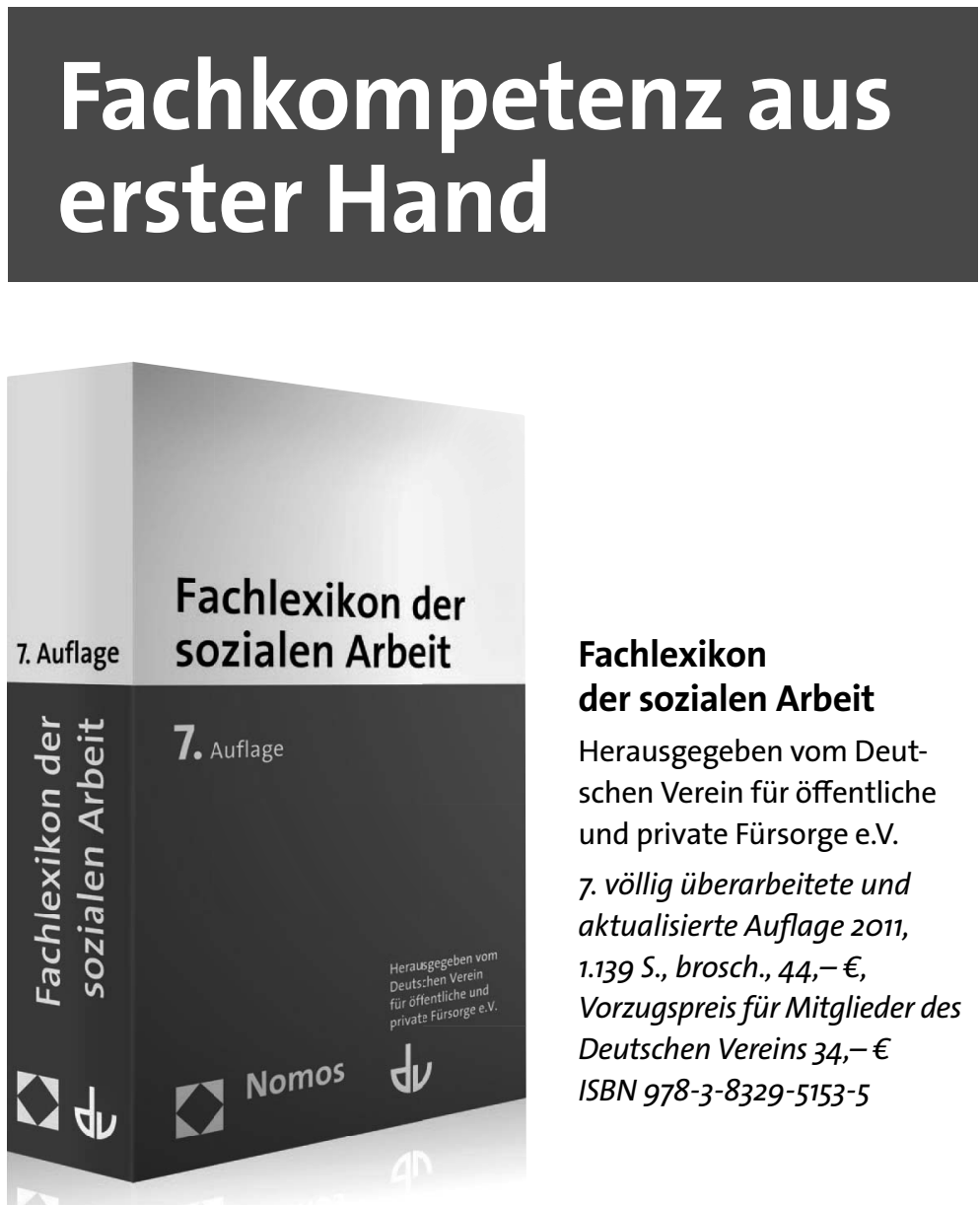

\section{Fachlexikon der sozialen Arbeit}

Herausgegeben vom Deutschen Verein für öffentliche und private Fürsorge e.V.

7. völlig überarbeitete und aktualisierte Auflage 2011, 1.139 S., brosch., 44,- $€$, Vorzugspreis für Mitglieder des Deutschen Vereins 34,- $€$ ISBN 978-3-8329-5153-5

Das „Fachlexikon“ ist das Standardwerk für die soziale Arbeit. Die aktuelle 7. Auflage umfasst über 1.500 übersichtlich strukturierte und teilweise neu systematisierte Stichwörter, deren Auswahl sich strikt nach den Bedürfnissen der sozialen Arbeit richtet.

Über 600 Autorinnen und Autoren aus Wissenschaft und Praxis der sozialen Arbeit berücksichtigen den neuesten Stand der verschiedenen Entwicklungen und Diskurse und werden damit dem pluralistischen Anspruch des Fachlexikons gerecht.

\section{Die Neuauflage}

- geht auf die Reformentwicklungen zu Hartz IV ein,

- stellt alle wichtigen fachlichen Entwicklungen, z.B. in der Familienpolitik (Stichworte „Elternzeit“, „Vereinbarkeit von Familien- und Erwerbsleben“) oder im Rahmen neuer Konzepte der sozialen Arbeit („,care“) dar,

verortet und umfasst stets klar die neuesten methodischen Ansätze sozialer Arbeit,

- nimmt Rücksicht auf die Lesegewohnheiten durch ein ausdifferenziertes System von ca. 700 Verweisstichwörtern sowie einem neu aufgenommenen Wortverzeichnis. Das einschlägige Stichwort wird rasch gefunden, Lektüreempfehlungen wie ein über 2.500 Titel umfassendes Gesamtliteraturverzeichnis geben sinnvolle Vertiefungshinweise.

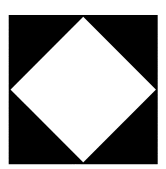

Bitte bestellen Sie im Buchhandel oder versandkostenfrei unter $\downarrow$ www.nomos-shop.de 